\title{
A Simplified Recognition Algorithm of Digital Planes Pieces
}

\author{
Mohammed Mostefa Mesmoudi \\ Department of Computer Science and Information Science (DISI) \\ Science University of Genoa \\ Via Dodecaneso, 35 -16146 Genoa (Italy) \\ mesmoudi@disi.unige.it
}

\begin{abstract}
Debled proposed an efficient algorithm for the recognition of rectangular digital planes pieces. However, uses, in some cases called strongly exterior cases, some validity criteria which are only sufficient but not necessary. In this paper we give necessary and sufficient conditions (including strongly exterior cases) to recognize pieces of digital planes. We build up a simplified form of Debled's algorithm. Furthermore, our approach is independent from the rectangular form of pieces considered by Debled.
\end{abstract}

\section{Introduction}

Let $\nu$ be a 18-connected bounded convex subset of $Z^{3}$ which injectively projects on a subset $\Pi$ of the plane $O x y$. Let $\omega$ be a positive integer, if there exist integers $a, b, c, \mu$ in $Z$ such that $\nu$ is the set of solutions of the double Diophantine inequality

$$
\left\{\begin{array}{l}
\mu \leq a x+b y+c z<\mu+\omega \\
(x, y) \in \Pi
\end{array}\right.
$$

then we say that $\nu$ is a piece of a digital plane $P(a, b, c, \mu, \omega)$ with characteristics $a, b, c$ and lower bound $\mu$. The number $\omega$ is called the arithmetic thickness of the plane. When $\omega=\sup (|a|,|b|,|c|)$, the plane $P(a, b, c, \mu, \omega)$ is called a naïve plane and denoted by $P(a, b, c, \mu)$ [1]. Let us consider a family $\left\{\nu_{t}\right\}_{t \in[\mu, \mu+\omega-1]}$ of real parallel planes $\nu_{t}$ defined by $a x+b y+c z=t$. The digital plane $\nu$ is the intersection of the above family with $Z^{3}$ that can be geometrically represented by a set of voxels as shown in Figure 1. Let us suppose that the greatest common divisor of $a, b, c$ is 1 . We say that $\nu$ is recognized if it contains sufficiently points to compute all the characteristics $a, b, c, \mu, \omega$. To this aim, it is sufficient that $\nu$ possesses four affinely independent points that generate the bounding real planes $\nu_{\mu}$ and $\nu_{\mu+\omega-1}$. The problem of studying pieces of digital naïve planes can be reduced to the case where $0 \leq a \leq b \leq c$. Indeed, the general case can be obtained from the former case by rotations and symmetries [3]. There is a wide literature on the problem of recognizing digital plane pieces. Kim and Rosenfeld showed in [6] that a digital surface is a piece of a naïve digital plane if and only

A. Braquelaire, J.-O. Lachaud, and A. Vialard (Eds.): DGCI 2002, LNCS 2301, pp. 404416 2002. (C) Springer-Verlag Berlin Heidelberg 2002 
if there exists a face of the convex hull of the surface such that the distance between the points of the surface and the plane that supports the face is less than 1 . They proposed an algorithm based on this property of complexity $O\left(p^{4}\right)$, where $p$ is the number of points on the surface. In 1991, Kim and Stojmenović [7] improved this algorithm to obtain another algorithm of complexity $O\left(p^{2} \log p\right)$. In the same year, Stojmenović and Tos̃ić 11] presented two other algorithms, the first with complexity $O($ plogp $)$ based on the construction of two convex hulls and the second with complexity $O(p)$ and based on linear programming in $3 D$. These algorithms have a low complexity but they are not incremental which is a drawback in application. Furthermore, the construction of the convex hull in $3 D$ is a delicate and expensive operation. In 1312 , Veelaert, relying on a generalization of a regularity property of digital straight lines introduced by Hung [5], developed a simple algorithm of complexity $O\left(p^{2}\right)$ which is satisfactory for small sets $(p \leq 100)$.

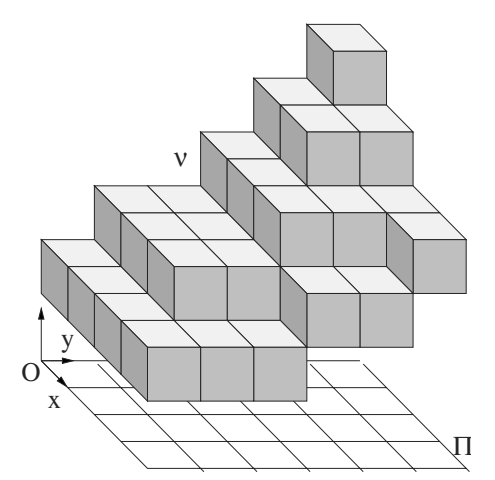

Fig. 1. The shape of a possible digital plane piece

The arithmetic definition of digital planes led by relation (1) was introduced for the first time by Reveilles in 1991, [10]. In 1995, Debled proposed, in her PhD thesis [3], an efficient algorithm for the recognition of rectangular pieces of digital planes. This algorithm has more advantages than the other algorithms quoted above. It uses a simple and intuitive geometric properties of digital planes. It has a quadratic complexity and is incremental. It consists in sweeping a piece by sections that are parallel to a co-ordinate plane. At the beginning, one fixes, for instance, $y=0$ and let $x$ vary. In this case, Debled's algorithm tries to recognize digital straight lines until $x$ reaches its maximum value in $\Pi$. Then, $y$ is incremented by 1 and $x$ varies again. In this case, Debled's algorithm tries to recognize pieces of digital planes. At each step the algorithm tries to compute the characteristics of the new plane. Three cases may occur

- If the added point $M(x, y)$ satisfies relation (1), then the same characteristics of the last recognized piece are kept.

- If the added point $M$ satisfies one of the following relations

$$
a x+b y+c z=\mu-1 \quad \text { or } \quad a x+b y+c z=\mu+c
$$


where $(a, b, c)$ is the normal vector of the last recognized piece, the point $M$ is said to be 1-exterior to the last piece. Debled conjectured that the new piece is recognized in a new plane. By means of other conjectures, it is possible to construct the new characteristics. All these conjectures have been checked by numerous examples. Mathematical proofs of these conjectures have been presented in [2,98]. The complexity of Debled's algorithm corresponding to this step is at most linear in the number of points on the piece, see 3 p.180.

- The third possibility is that $M$ satisfies one of the following inequalities

$$
a x+b y+c z<\mu-1 \quad \text { or } \quad a x+b y+c z>\mu+c
$$

In this case we say that $M$ is strongly $k$-exterior, where $k$ is equal to $\mu-$ $(a x+b y+c z)$ or $(a x+b y+c z)-\mu-c+1$ respectively. The new piece belongs to a digital plane if $M$ is not too distant from the piece. Debled gave three validity criteria to be checked for the new piece to conclude its flatness. These criteria are sufficient but not necessary, see [3] p. 172.

In this paper we present necessary and sufficient conditions to recognize pieces of digital planes. We obtain a new algorithm that simplifies and generalizes Debled algorithm. Its complexity is at most quadratic since we exploit, for each added point, only the part of Debled's algorithm used for 1-exterior case.

Next section introduces some fundamental notions of digital planes that will be used throughout the paper. Section 3 surveys our fundamental results on 1exterior case. Section 4 addresses the problem of recognition in strongly exterior case and two recognition theorems are presented. Section 5 describes our algorithm that simplifies Debled's one, whereas Section 6 explains it by an example.

\section{Background}

Let us begin by giving the definitions of some notions related to digital planes that we will use in this paper. Let $M(x, y, z)$ be a point of a digital plane $P(a, b, c, \mu)$, the quantity $r(M)=a x+b y+c z$ is called the remainder of $M$ with respect to $P$. The Diophantine inequality(1) allows us to define $P(a, b, c, \mu)$ as the set of points $(x, y, z) \in Z^{3}$ such that $\frac{\mu-a x-b y}{c} \leq z<\frac{\mu-a x-b y}{c}+1$. Since the plane projects injectively on $O x y$, we can represent $P(a, b, c, \mu)$ in $O x y$ by level lines corresponding to values of $z$. We can also represent the plane $P(a, b, c, \mu)$ by the remainder of its points. In Figure 2 we combine the remainder and level lines representations to represent $P(9,13,21,0)$ on plane $O x y$. The real plane defined by $r(a, b, c)(M)=k$ is called the plane of index $k$. The plane of index $\mu$ is called lower leaning plane of $P$ and the plane of index $\mu+c-1$ is called upper leaning plane of $P$. We denote them by $(P i)$ and $(P s)$ respectively.

A piece of plane is a convex subset of voxels of a naïve digital plane. A piece of a digital plane is said to be recognized if it possesses four leaning points that satisfy one of the following two cases:

- Three upper (resp. lower) leaning points and one lower (resp. upper) leaning point. This configuration is referred to as CAS3.1, see figure 2(a). 
- Two upper leaning points and two lower leaning points. This configuration is refereed to as CAS2.2, see figure 2(b).

\begin{tabular}{|c|c|c|c|c|c|c|c|c|c|c|}
\hline 18 & 6 & 15 & 3 & 12 & 0 & 9 & 18 & 6 & 15 \\
\hline 5 & 14 & 2 & 11 & 20 & 8 & 17 & 5 & 14 & 2 \\
\hline 13 & 1 & 10 & 19 & 7 & 16 & 4 & 13 & 1 & 10 \\
\hline 0 & 9 & 18 & 6 & 15 & 3 & 12 & 0 & 9 & 18 \\
\hline
\end{tabular}

(a)

\begin{tabular}{|c|c|c|c|c|c|c|c|c|c|c|}
15 & 18 & 21 & 2 & 5 & 8 & 11 & 14 & 17 & 20 \\
\hline 10 & 13 & 16 & 19 & 0 & 3 & 6 & 9 & 12 & 15 \\
\hline 5 & 8 & 11 & 14 & 17 & 20 & 1 & 4 & 7 & 10 \\
\hline 0 & 3 & 6 & 9 & 12 & 15 & 18 & 21 & 2 & 5 \\
\hline
\end{tabular}

(b)

Fig. 2. (a) CAS3.1. The remainder and level lines representation of a piece of $P(9,13,21,0)$ that contains three lower leaning points and one upper leaning point. (b) CAS2.2. The remainder and level lines representation of a piece of $P(3,5,22,0)$ that contains two lower leaning points and two upper leaning points

When the added point $M$ is $k$-exterior, many geometric constructions are built and key voxels are extracted. These geometric constructions depend on leaning polygons and their positions with respect to $M$. These leaning polygons are defined as follows:

- If $r(M)<\mu$, we call $(P S)$ the convex hull of the upper leaning points in the piece and we call it the upper leaning polygon. We define, in this case, the convex polygon of pivots $C V P$ to be the upper leaning polygon $P S$.

In the same way, we call $P I$ the lower leaning polygon. In this case, we define the convex polygon of antipodes $C V A$ to be the lower leaning polygon $P I$. Thus, we have $C V P=P S$ and $C V A=P I$.

- If $r(M) \geq \mu+c$, the upper leaning polygon $P S$ is called the convex polygon of antipodes $C V A$ and the lower leaning polygon $P I$ is called the convex polygon of pivots $C V P$. We have $C V A=P S$ and $C V P=P I$.

In figure 3(a) we give an example of the convex polygons of pivots and antipodes for a recognized piece in the plane $P(5,6,7,-1)$.

The polygonal line of pivot vectors $L$ is constructed depending on the added $k$-exterior point and its associated convex polygon of pivots. All constructions are realized on the projections in the plane Oxy. Four cases are possible:

1. The $C V P$ is reduced to one point. In this case, the polygonal line of pivot vectors $L$ is reduced to this point.

2. The $C V P$ is formed by points that are not all collinear. In this case, the polygonal line $L$ of pivot vectors is composed by points of the $C V P$ such that their projections in the plane $O x y$ are located on the part of the boundary of the convex hull of the $C V P$ projection that disappears when the point $M$ is added, see Figure 3(b).

3. The $C V P$ is composed by collinear points in the plane $O x y$ : 


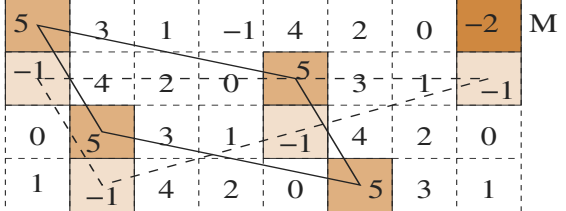

(a)

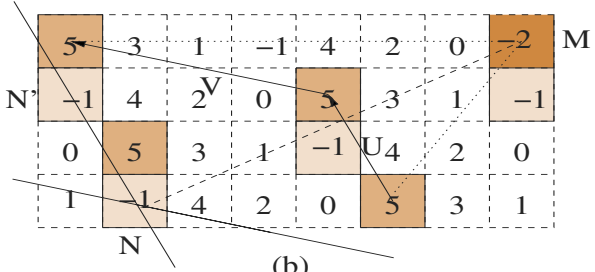

(b)

Fig. 3. The point $M$ is 1-exterior of remainder -2 . In (a) the parallelogram in bold is the convex of pivot points $C V P$ and the dashed triangle is the convex polygon of antipodes $C V A$. In (b) $U \cup V$ form the polygonal line of pivot vectors $L$, point $N$ is an antipode for both pivot vectors $U$ and $V$, It is separating for the vector $U$. Point $N^{\prime}$ is an antipode for the vector $U$ only.

- If the projection of $M$ is collinear with the projected points of the $C V P$, then the polygonal line $L$ of pivot vectors is reduced to the nearest point of the $C V P$ to $M$.

- If the projection $M$ is not collinear with the projected points of the $C V P$, then $L$ is equal to all points of the $C V P$.

An antipode $A$ of a pivot vector $V$ associated to a $k$-exterior point $M$ is a summit of the $C V A$ that has the maximal distance, among all points of the $C V A$, from the line directed by $V$ and containing $M$. Furthermore, if the end points of $V$ are separated by the line $M A$, then $A$ is called a separating antipode.

\section{The 1-Exterior Case}

Let $S$ be a recognized piece of the plane $P(a, b, c, \mu)$ and $M$ an added point to $S$. If the remainder of $M$ with respect to $P(a, b, c, \mu)$ is between $\mu$ and $\mu+c-1$, then $S^{\prime}=S \cup\{M\}$ is still recognized in the same plane. If the point $M$ is 1exterior, Debled's conjectures 314 assure the existence of a new plane in which $S^{\prime}$ is recognized. Furthermore, following the shape of the polygonal line of pivot vectors, these conjectures give methods to construct the new characteristics $A \leq$ $B \leq C$ such that $C$ is the smallest for all possible planes that contain $S^{\prime}$. These conjectures have been mathematically proven in recent works [289.

In fact, we prove in 8 , the following key theorem that assures the existence of planes that contain $S^{\prime}$. We gave also a method to construct the smallest characteristics.

Theorem $([8])$. Let $S$ be a recognized piece of the plane $P(a, b, c, \mu)$ and $M\left(x_{0}\right.$, $\left.y_{0}, z_{0}\right) \in Z^{3}$ be a point such that $S^{\prime}=S \cup\{M\}$ is convex. If $M$ is 1 -exterior to $S$, then there exists $\left(A, B, C, \mu^{\prime}\right) \in Z^{4}$ with $A \wedge B \wedge C=1$ such that $S^{\prime}=S \cup\{M\}$ is a piece of the plane $P\left(A, B, C, \mu^{\prime}\right)$.

The proof of this theorem provides two necessary and sufficient conditions on the 
choice of points that allow the construction of the new base. These conditions are:

$$
\beta_{2}\left(x_{2}-x_{0}\right)-\alpha_{2}\left(y_{2}-y_{0}\right)=c,
$$

where $\left(x_{2}, y_{2}\right),\left(x_{1}, y_{1}\right)$ and $\left(\alpha_{2}, \beta_{2}\right)$ are respectively the projections on $O x y$ of a pivot point $M_{2}$, an antipode $M_{1}$ and a vector $V_{2}$ formed by $M_{2}$ and another pivot point.

$$
d_{m}-(\mu+c-1)+\frac{h_{2}}{H} \leq \frac{\epsilon_{m} h_{m}}{H} \leq d_{m}-(\mu-1) \forall m \in S,
$$

where $\epsilon_{m}$ is the sign of $\beta_{2}\left(x-x_{0}\right)-\alpha_{2}\left(y-y_{0}\right)$, the numbers $H, h_{2}$ et $h_{m}$ are the Euclidean distances between the points $M_{1}, M_{2}$ and $m$ respectively and the real line directed by $V_{2}$ and containing $M$.

\section{The Strongly Exterior Case}

Let us now suppose that $M$ is strongly exterior to the piece $S \subset P(a, b, c, \mu)$. This implies that there exists an integer $P \geq 2$ such that $M$ satisfies one of the following equalities: $a x_{0}+b y_{0}+c z_{0}=\mu-P$ (i.e, $M$ is located under the piece $S$ ) or $a x_{0}+b y_{0}+c z_{0}=\mu+c-1+P$ (i.e, $M$ is located over the piece $S$ ). An axial rotation of the plane by $\pi$ reverses the positions of its points. Points located under the plane get over it and vice versa. The characteristics of the plane remain unchanged. This fact allows us to reduce our study to one case, say $M$ being located under the plane. All relations we will obtain are still valid in the case where $M$ is located over the plane. The following lemma is a direct consequence of convexity.

Lemma 1. Let $S$ be a piece of a discrete plane $P(a, b, c, \mu)$ and $M\left(x_{0}, y_{0}, z_{0}\right)$ a point such that $S^{\prime}=S \cup\{M\}$ is convex. If $M$ is strongly $P$-exterior then $P \leq c$.

Proof. Suppose that the remainder of $M$ is $r(M)=\mu-P$ and let consider the point $M^{\prime}\left(x_{0}, y_{0}, z_{0}+1\right)$. The remainder of $M^{\prime}$ is $r\left(M^{\prime}\right)=\mu-P+c$. If $r\left(M^{\prime}\right)<\mu$, then $M$ is disconnected from $S$ and $S^{\prime}$ is not convex. Hence, $r\left(M^{\prime}\right) \geq \mu$ which gives $P \leq c$.

\subsection{Recognition Theorems}

The piece $S$ is bounded by two real leaning planes of indices $\mu$ and $\mu+c-1$. When the added point $M$ is exterior to $S$, located for instance under $S$, then, in order to geometrically contain $S^{\prime}=S \cup\{M\}$ in another digital plane, one should lean the plane of index $\mu$ in a suitable direction until $M$ is reached. This latter plane becomes the lower leaning plane of the "eventual" digital plane we are looking for. Then let us consider another plane located over $S$, tangent to $S$ and parallel to the new lower leaning plane. The distance between these two planes should be less than or equal to 1; otherwise we don't get a digital plane. 
The operation of leaning planes can be done by revolving on some parallel straight lines all planes of indices between $\mu$ and $\mu+c-1$. If the line, on which pivots the plane of index $\mu+c-1$, is too distant from $M$, then we may lose some upper leaning points (i.e., points of $C V P$ ) located between this line and $M$ after the rotation, see Figure 4(a). Thus, this line should be chosen as close as possible to the point $M$. In the same way, the rotation of the lower leaning plane should not exclude the lower leaning points. Therefore, the line on which we pivot this plane should be chosen as far as possible from $M$ so that all points of the $C V A$ will be located between this line and $M$, see Figure 4(b). This explains the importance of polygonal lines of pivots and antipodes defined above.

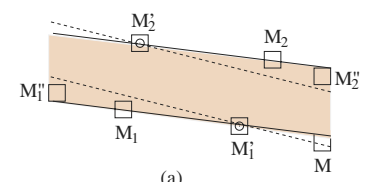

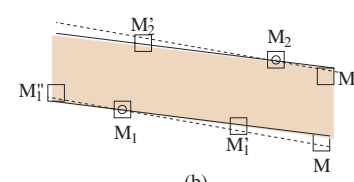

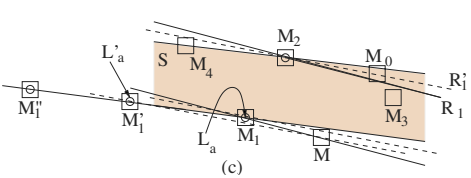

Fig. 4. Profile view: Axes of rotation are shown by $(\odot)$, the result is delimited by the dashed lines. In the 3D-space points, as $M, M^{\prime \prime}{ }_{2}$, are not piled up. In (a) the rotation excludes leaning points $M_{1}, M_{2}$ and eventual internal points as $M^{\prime \prime}{ }_{1}$ and $M{ }_{2}$. In (b) the rotation does not exclude any point of the piece. In (c) the rotation $R_{1}$ excludes point $M_{0}$, while rotation $R_{1}^{\prime}$ includes all points of $S$, its axe contains $M_{1}^{\prime}$. Leaning point $M_{4}$ becomes an internal point.

After this operation $M$ become naturally a lower leaning point of the new digital plane that contains $S^{\prime}$. In practice, this operation does not always give a recognized piece of a digital plane. This is due to the fact that when $M$ is too distant from $S$, the rotation of the above upper (resp. lower) leaning plane may excludes some points (i.e., their remainders are beyond the bounds given by relation 1). To palliate to this problem one can choose the lines, on which the leaning planes pivot, outside the piece. But the result we obtain is that either the new digital plane is not naïve (the distance between the resulting leaning planes is greater than 1) or the piece is not recognized (the number of the new leaning points in the piece is strictly inferior to 4). Thus, to get a satisfactory solution we have to carefully handle the operation of leaning planes. We proceed as follows:

We rotate the upper leaning plane, by a suitable angle, as explained bellow on the nearest line $L_{p}$ to $M$. We rotate the lower leaning plane on the most far line $L_{a}$ from $M$ which is parallel to $L_{p}$ (in the sense that it is directed by the same vector of $L_{p}$ ) and contains a leaning lower point in $S$. These lines strongly depend on the polygonal line of pivots $L$ and the polygonal line of antipodes $L^{\prime}$. Since each of $L$ and $L^{\prime}$ contains no more than two independent vectors [39] then $L_{p}$ and $L_{a}$ are chosen in one of the following configurations:

1. If the polygonal line of pivots $L$ is not reduced to a point and if there exists a separating antipode $M_{1}$, then $L_{p}$ is the line containing vector $V_{2}$ of $L$ which 
is separated by $M_{1}$. We take $L_{a}$ as the line directed by $V_{2}$ and containing $M_{1}$.

2. If $L$ is reduced to one point, then, since $S$ is recognized, four leaning points exist that define the normal vector of $S$ and its thickness. These points define at least two independent vectors. Since the leaning points on $L$ are collinear, then other leaning points (antipodes) exist on the CVA that define a transverse direction to $L$. Among antipodes on this transverse direction we take the line $L_{a}$ which contains the most far antipode from $M$. Then we define line $L_{p}$ to be parallel to $L_{a}$ and to contain the nearest pivot point to $M$ (which is, in this case, equal to $L$ ).

3. If $L$ is not reduced to a point and there is no separating antipode, then we proceed in the same way as in the previous case to define $L_{a}$ and $L_{p}$.

The angle of rotation is chosen such that

(i) if $M$ is reached without excluding any point of $S$ (i.e., all points of $S$ still satisfy a relation of type (11) after the rotation), then this rotation, say $R_{1}$, gives a new naïve digital plane in which the piece $S^{\prime}$ is recognized.

(ii) if the previous rotation excludes a point only on $L_{p}$, since all possible points on $L_{p}$ should have the same remainder, then all these points are excluded. Thus $M$ is too distant. To keep the points of $L_{p}$ in the new plane we have to stop rotation $R_{1}$ before reaching $M$. In this case, $L_{p}$ and $L_{a}$ are still the pivots and antipodes lines of the new plane and there is no way to reach $M$ by a rotation. We note that, in this case, $M$ becomes less strongly exterior but never 1-exterior because in this later case the piece becomes recognizable which is impossible.

(iii) if the previous rotation $R_{1}$ excludes a point $M_{2}$ of $L_{p}$ (or $L_{a}$ ) after having reached a point $M_{0}$ of remainder $\mu+c-2($ or $\mu+1)$, then we reduce the angle of rotation until $M_{0}$ is reached. Let $R_{1}^{\prime}$ be the corresponding rotation. Algorithmically, this can be done by moving line $L_{a}$ to the next line $L_{a}^{\prime}$ that is parallel to $L_{a}$, contains antipodes, and its distance to $M$ is bigger than $L_{a}$ 's one, see Figure $4(\mathrm{c})$.

The leaning points of $L_{a}$ and $L_{p}$ always remain leaning points of the new digital plane, the other leaning points become internal points in general, and $M_{0}$ becomes a new leaning point. Thus, we get enough leaning points so that the piece $S$ still be recognized in the new digital plane.

- If point $M$ becomes 1-exterior for the new characteristics, then Debled algorithm allows us to recognize the piece $S^{\prime}$ in another digital plane.

- If point $M$ remains strongly exterior, then we have to apply a new rotation. But in this case, point $M_{2}$ will be excluded and we are in situation (ii). Therefore, piece $S \cup\{M\}$ can not be recognized in a digital plane.

(iv) If rotation $R_{1}$ excludes a point $M_{0}$ of remainder $\mu+c-2($ or $\mu+1)$ and points of $L_{p}$ and $L_{a}$ remain included by $R_{1}$ then we have to stop the rotation before excluding $M_{0}$. This corresponds to an extremal position and piece $S \cup\{M\}$ can not be recognized. 
All these cases imply that strongly exterior cases can be recognized only through 1-exterior cases. This proves our first theorem of recognition. However, before doing any rotation, there is an analytic way to check what kind of situation we may have. This analytic way is given by condition 5 in the second theorem of recognition.

Theorem 1. Let $S$ be a recognized piece of a digital plane $P(a, b, c, \mu)$ and $M \in$ $Z^{3}$ be a point such that $S^{\prime}=S \cup\{M\}$ is convex. Suppose that $M$ is strongly $P$ exterior $(P \geq 2)$. Then there exists at most one digital naïve plane in which $S$ is recognized and admits $M$ as a strongly q-exterior for some integer $2 \leq q<P$. When a such number $q$ exists, then $S^{\prime}$ cannot be recognized in a naïve digital plane.

Theorem 2. Let $S$ be a recognized piece of a digital plane $P(a, b, c, \mu)$ and $M\left(x_{0}, y_{0}, z_{0}\right) \in Z^{3}$ be a point such that $S^{\prime}=S \cup\{M\}$ is convex. Suppose that $M$ is strongly $P$-exterior $(P \geq 2)$. Let $M_{2}\left(x_{2}, y_{2}, z_{2}\right)$ be a point on the polygonal line of pivots and $V_{2}\left(\alpha_{2}, \beta_{2}, \gamma_{2}\right)$ a vector based on $M_{2}$ and located either on the polygonal line of pivots if it is not reduced to a point or parallel to the polygonal line of antipodes. Let $V_{1}\left(\alpha_{1}, \beta_{1}, \gamma_{1}\right)$ be a vector linking $M$ to an antipode $M_{1}$ sufficiently distant from $M$ in order for it to exceed at least the polygonal line of antipodes.

Let $A=\beta_{1} \gamma_{2}-\beta_{2} \gamma_{1}, B=\alpha_{2} \gamma_{1}-\alpha_{1} \gamma_{2}, C=\alpha_{1} \beta_{2}-\alpha_{2} \beta_{1}$ and $\left.k=A \wedge B \wedge C\right)$. Then piece $S$ belongs to a digital plane $P\left(\frac{A}{k}, \frac{B}{k}, \frac{C}{k}, \mu^{\prime}\right)$ such that $M$ is a leaning point $(q=0)$ or 1-exterior $(q=1)$ if and only if $M_{1}$ is arbitrarily chosen on a line, among at most three parallel lines, directed by $V_{2}$ such that the following relations are satisfied:

$$
\begin{gathered}
0 \leq A \leq B \leq C \\
P\left(\beta_{2}\left(x_{2}-x_{0}\right)-\alpha_{2}\left(y_{2}-y_{0}\right)\right)-C(P-1)=k c(1-q), \\
\frac{d_{m}-\mu-c+P}{P}+\frac{k(1-q) c}{\left|V_{2}\right| H} \leq \frac{\epsilon_{m} h_{m}}{H} \leq \frac{d_{m}-\mu+P}{P} ; \forall m \in S,
\end{gathered}
$$

with $q=0$ or 1 and $H$ (resp. $h_{m}$ ) being the height of point $M_{1}$ (resp. $m$ ) to the real line passing by $M$ and directed by $V_{2}$.

The number of lines that may contain $M_{1}$ is 1 if $k<P$ and at most 3 if $k=P$.

\section{Remarks.}

1. In the algorithm that we propose, only relations (4) and (5) are needed explicitly, relation (6) serves mathematically to prove Debled's conjectures and explains how to choose $M_{1}$ between all antipodes.

2 . We note that if in theorem 2 we assume that $P$ can take the value 1 , relations (4), (51) and (6) become equivalent to those quoted in the theorem of the previous section. Following lemma 2 below, the number $k=A \wedge B \wedge C$ divides $P$ which is assumed to be 1 here. This fact is also true for the 1 -exterior case. Thus theorem 2 includes the 1-exterior case. 
Proof Idea. Let us suppose that the added point $M$ is located under plane $P(a, b, c, \mu)$. The Point $M$ satisfies the relation $a x_{0}+b y_{0}+c z_{0}=\mu-P$. Let $M_{1}$ be a lower leaning point of $P(a, b, c, \mu)$ and let $V_{1}=\overrightarrow{M M_{1}}=:\left(\alpha_{1}, \beta_{1}, \gamma_{1}\right)$ and $(A, B, C)=: V_{1} \wedge V_{2}$. Vector $V_{1}$ satisfies the relation $a \alpha_{1}+b \beta_{1}+c\left(\gamma_{1}-\frac{P}{c}\right)=0$. Suppose that $V_{2}$ is linearly independent from $V_{1}^{\prime}\left(\alpha_{1}, \beta_{1}, \gamma_{1}-\frac{P}{c}\right)$. The vectorial product $V_{1}^{\prime} \wedge V_{2}=\left(A^{\prime}, B^{\prime}, C^{\prime}\right)$ is a rational multiple, say $\lambda$, of $N(a, b, c)$. The numbers $A^{\prime}, B^{\prime}, C^{\prime}$ are given by:

$$
A^{\prime}=A+\beta_{2} \frac{P}{c}=\lambda a ; \quad B^{\prime}=B-\alpha_{2} \frac{P}{c}=\lambda b ; \quad C^{\prime}=C=\lambda c .
$$

We can always assume (by considering $-V_{2}$ in place of $V_{2}$ ) that $0 \leq A^{\prime}$. Since $0 \leq a \leq b \leq c$, then $0 \leq A^{\prime} \leq B^{\prime} \leq C^{\prime}$ which is equivalent to $0 \leq \bar{A}+\beta_{2} \frac{P}{c} \leq$ $B-\alpha_{2} \frac{P}{c} \leq C$. If we increase the length of $V_{1}$, then the numbers $A^{\prime}, B^{\prime}, C^{\prime}$ also increase, and so does the distance between each other. Note that the quantities $\beta_{2} \frac{P}{c}$ and $\alpha_{2} \frac{P}{c}$ do not change when we increase the length of $V_{1}$. Let us take $V_{1}$ sufficiently long so that

$$
0 \leq A \leq B \leq C
$$

By dividing $A, B, C$ by their greater common divisor, we obtain the characteristics of the eventual new plane which may contain the piece $S^{\prime}=S \cup\{M\}$.

Lemma 2. If the numbers $\alpha_{2}, \beta_{2}$ and $\gamma_{2}$ are prime together $\left(\alpha_{2} \wedge \beta_{2} \wedge \gamma_{2}=1\right)$, then $P G C D(A, B, C)=A \wedge B \wedge C$ divides number $P$.

Let us search conditions under which there exists $\mu^{\prime}$ such that the point $M$ is $q$-exterior to $S \subset P\left(A, B, C, \mu^{\prime}\right)$, with $q \in\{0,1\}$. This means that for all $m(x, y, z) \in S^{\prime}$ we have:

$$
\left\{\begin{array}{l}
\mu^{\prime} \leq A x+B y+C z<\mu^{\prime}+C \\
A x_{0}+B y_{0}+C z_{0}=\mu^{\prime}-P+q \quad \text { where } \quad q=0 \text { or } 1
\end{array}\right.
$$

For all $m(x, y, z) \in S \subset P(a, b, c, \mu)$ we have $\mu \leq a x+b y+c z \leq \mu+c-1$. The added point $M$ satisfies the relation $a x_{0}+b y_{0}+c z_{0}=\mu-P$. Then $\mu-P \leq$ $a x+b y+c z \leq \mu+c-1$, for all points of $S^{\prime}$.

By multiplying all members with $\lambda$ we obtain:

$$
\lambda(\mu-P)-P\left(\frac{\beta_{2}}{c} x-\frac{\alpha_{2}}{c} y\right) \leq A x+B y+C z \leq \lambda(\mu-1)-P\left(\frac{\beta_{2}}{c} x-\frac{\alpha_{2}}{c} y\right)+C .
$$

Let us take $k=P G C D(A, B, C)$. By dividing the previous inequality with $k$ we obtain:

$\lambda "(\mu-P)-P^{\prime \prime} \frac{\left(\beta_{2} x-\alpha_{2} y\right)}{c} \leq A^{\prime \prime} x+B " y+C^{\prime \prime} z \leq \lambda^{\prime \prime}(\mu-1)-P^{\prime \prime} \frac{\left(\beta_{2} x-\alpha_{2} y\right)}{c}+C^{\prime \prime}$, where $P^{\prime \prime}=\frac{P}{k}, \quad A^{\prime \prime}=\frac{A}{k}, \quad B^{\prime \prime}=\frac{B}{k}, C^{\prime \prime}=\frac{C}{k}$ et $\lambda "=\frac{\lambda}{k}=\frac{C "}{c}$.

Let us take $\mu(x, y)=\lambda^{\prime \prime}(\mu-P)-P^{\prime \prime} \frac{\left(\beta_{2} x-\alpha_{2} y\right)}{c}$. Then for all points in $S^{\prime}$ we have: $\mu(x, y) \leq A^{\prime \prime} x+B " y+C " z \leq \mu(x, y)+C "{ }^{\prime \prime}+\lambda^{\prime \prime}(P-1)$.

Using the facts that $M$ is $P$-exterior and should become $q$-exterior and that $M_{2}$ (one of the end points of vector $V_{2}$ ) is an upper leaning point of pieces $S$ and $S^{\prime}$, we obtain the condition 


$$
P\left[\beta_{2}\left(x_{2}-x_{0}\right)-\alpha_{2}\left(y_{2}-y_{0}\right)\right]-C(P-1)=k c(1-q) .
$$

This condition expresses the position of points $M_{2}, M_{2}^{\prime}$ and $M_{1}$ with respect to $M$. Now let us search in what region we can choose $V_{1}$ so that surface $S$ will be a recognized piece of the digital plane of parameters $\left(A ", B\right.$ ",$C$ ", $\left.\mu\left(x_{0}, y_{0}\right)+q\right)$ with $M$ a $q$-exterior, where $q=0$ or 1 . The former double inequality can be transformed to get $\frac{d_{m}-\mu-c+P}{P}+\frac{k c(1-q)}{P C} \leq \frac{\beta_{2}\left(x-x_{0}\right)-\alpha_{2}\left(y-y_{0}\right)}{\beta_{2} \alpha_{1}-\alpha_{2} \beta_{1}} \leq \frac{d_{m}-\mu+P}{P}$. This expression can be expressed in terms of heights by:

$$
\frac{d_{m}-\mu-c+P}{P}+\frac{k(1-q) c}{\left|V_{2}\right| H} \leq \frac{\epsilon_{m} h_{m}}{H} \leq \frac{d_{m}-\mu+P}{P}
$$

with $d_{m} \in[\mu-P, \mu+c-1]$. It is the condition that all points $m(x, y, z) \in S^{\prime}$ should satisfy so that $S^{\prime}$ will be a recognized piece in the digital plane of characteristics $\left(A ", B ", C ", \mu^{\prime}=\mu\left(x_{0}, y_{0}\right)\right)$. By studying the sign of the first member of relation (5), we can show that this relation can be satisfied at most by three lines directed by $V_{2}$ and containing antipodes.

\section{Simplified Recognition Algorithm}

The algorithm that we describe in this section directly derives from the discussion and the proofs of the two previous theorems. It uses only the part of Debled's algorithm corresponding to 1-exterior case with small modifications to recognize the general case of rectangular pieces. The complexity of the simplified algorithm decreases to become at most quadratic in the number of points in the piece. Following the necessary and sufficient conditions quoted in theorem 2, the simplified algorithm becomes completely decidable where the validity criteria given by Debled fail in the strongly exterior case. These two advantages are added to the other advantages of Debled's algorithm.

The algorithm begin by sweeping the piece to be recognized following sections parallel to one co-ordinate plane, $O x z$ for instance, by successively adding voxels. At the beginning we initialize $y=0$ and we let $x$ vary in its interval of definition. At each added voxel the algorithm tries to recognize a piece of a digital straight line. When all values of $x$ are considered we increment $y$ by 1 and we let $x$ sweep again over all its possible values. At each step the algorithm tries to recognize a piece in a digital plane and compute its characteristics. Three cases are possible:

1. If the added point $M$ satisfies the double inequality (1) for the plane constructed before adding $M$, then we keep the same characteristics and the updated piece is still recognized in this plane with the same leaning points with a possible addition of $M$.

2. If the added point is 1-exterior to the plane, then we apply Debled's algorithm to recognize the new piece. This step consists of computing polygonal pivots and antipodes lines to determine the vector $V_{2}$ which satisfies relation (2), and also search an adequate antipode $M_{1}$ that satisfies (3)).

3. In the third case, if the added point is strongly exterior, we search the polygonal line of pivots and antipodes and then check relation (5). If this relation 
is not satisfied for any point $M_{2}$ on the polygonal line of pivots, then the piece is not recognizable. If relation (5) is satisfied for some $q$ different from 0 and 1 then the piece is not recognizable. If relation (5) is satisfied for $q=0$, then we apply 1-exterior part of Debled's algorithm. In this case the piece is recognized with $M$ as a leaning point. If relation (5) is satisfied for $q=1$, then we again apply Debled's algorithm for the 1-exterior case at most three times to decide the recognition of the piece. Note that in this case, the choice of the antipode $M_{1}$ is modified from the choice given by Debled. In the first running of the algorithm, point $M_{1}$ is taken as in Debled's algorithm. If $M$ becomes 1-exterior, then we apply Debled's algorithm once more and the piece is recognized. At this point, if after one run there are some points which are excluded, then we choose the antipode $M_{1}$ on the following line proposed by theorem 2 ; if the point $M$ becomes 1-exterior, then we are done. If there are some points which are excluded then we take $M_{1}$ on the last line proposed by the theorem and the point becomes 1-exterior. Usually, only two lines are sufficient.

\section{Example}

In Figure 5(a), we represent a recognized piece $S$ of the plane $P(18,21,23,0)$. The added point $M(3,5,-7)$ is 2-exterior of remainder $r(M)=2$. The nearest pivot point to $M$ is $M_{2}(4,2,-4)$. The polygonal line of antipodes contains only two points $M_{1}(0,0,0)$ and $M_{1}^{\prime}(8,3,-9)$. The vector $V_{2}$ is then $(8,3,-9)$ and $V_{1}=$ $(-3,-5,7)$. The vectorial product of $V_{1}$ and $V_{2}$ gives $(A, B, C)=(24,29,31)$. With this choice relation (6) is not satisfied. Take for instance the point $m=$ $(9,0,-7)$. The quantity $\frac{h_{m}}{H}=\frac{\beta_{2}\left(x-x_{0}\right)-\alpha_{2}\left(y-y_{0}\right)}{C}=\frac{58}{31}=1,86$ is greater than $\frac{d_{m}-\mu+P}{P}=3 / 2=1,5$. Let us take the antipode located on the line directed by $V_{2}$ and that comes just after $V_{1}$. Let $M{ }_{1}(5,-1,-3)$ be a such antipode. The point $M "{ }_{1}$ is located outside the piece $S$. In this case vector $V_{1}$ is $\overrightarrow{M{ }_{1} M}=$ $(2,-6,4)$. The vectorial product $V_{1}$ with $V_{2}$ is $(42,50,54)=2(21,25,27)$. Note that $P G C D=k=2$ divides $P=2$.

The quantity $P\left(\beta_{2}\left(x_{2}-x_{0}\right)-\alpha_{2}\left(y_{2}-y_{0}\right)\right)-C(P-1)$ is equal to $2(3(4-3)-$ $8(2-5))-54(2-1)=54-54=0$. Moreover, for $q=1$ we obtain $k c(1-q)=0$. Thus, relation (5) is satisfied for $q=1$. We can check that all points of $S$ satisfy relation (6). For $m(9,0,-7)$, which did not satisfy (6) for the first basis, we get $\frac{h_{m}}{H}=\frac{58}{54}=1,07$ which is small to $\frac{d_{m}-\mu+P}{P}=3 / 2$ The value of $\mu^{\prime}=\mu\left(x_{0}, y_{0}\right)+q$ is $-1+1=0$. Piece $S$ is recognized in the plane $P(21,25,27,0)$, see Figure 5 (b).

Thus, we return back to 1-exterior case. The polygonal line of pivots is reduced to the point $M_{2}(4,2,-4)$. The polygonal line of antipodes contains two points $M_{1}(0,0,0)$ and $M_{1}^{\prime}(9,0,-7)$. Vector $V_{2}$ is then equal to $\overrightarrow{M_{1} M_{1}^{\prime}}(9,0,-7)$ and $V_{1}=\overrightarrow{M_{1} M}=(-3,-5,7)$. The vectorial product of $V_{1}$ with $V_{2}$ gives $(35,42,45)$. Piece $S^{\prime}=S \cup\{M\}$ becomes recognized in the plane $P(35,42,45,0)$ with at l;east three lower leaning points $M, M_{1}, M_{1}^{\prime}$ and at least one upper leaning point $M_{2}$, see Figure 5 (c). 

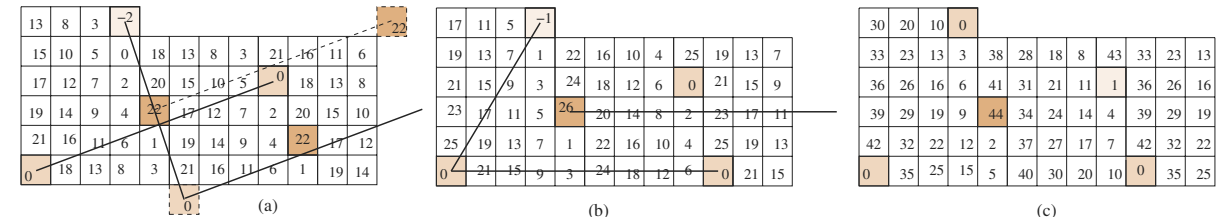

Fig. 5. In (a), piece $S$ is a recognized in the plane $P(18,21,23,0)$. In (b) piece $S$ is recognized in the plane $P(21,25,27,0)$ and $M$ is 1-exterior. In (c), piece $S^{\prime}$ is recognized in the plane $P(35,42,45,0)$

\section{References}

[1] E. Andrès. Cercles discrets et rotations discrètes. PhD thesis, Louis Pasteur University, December 1992.

[2] Y. Boukhatem. Sur la reconnaissance des plans discrets. Master Thesis, Mostaganem University, April 2001.

[3] I. Debled-Rennesson. Etude et Reconnaissance des Droites et Plans Discrets. $\mathrm{PhD}$ thesis, Louis Pasteur University, October 1995.

[4] I. Debled-Rennesson and J.P. Reveilles. Incremental algorithm for recognizing pieces of digital planes. In Spie's Internat. Symp. on Optical Science, Engeneering and Instrumentation, Technical conference Vision Geometry 5, Denver, USA, Aug 1996.

[5] S. H. Hung. On the straightness of digital arcs. In IEEE Transactions on Pattern Analysis and Machine Intelligence, volume PAMI 7, pages 203-215, 1985.

[6] C.E. Kim and A. Rosenfeld. Convex digital solids. In IEEE Transactions on Pattern Analysis and Machine Intelligence, volume PAMI 6, pages 639-645, 1984.

[7] C.E. Kim and I. Stajmenović. On the recognition digital planes in threedimensional space. In North-Holland, editor, Pattern Recognition Letters, volume 12, pages 665-669, 1991.

[8] M. M. Mesmoudi and I. Debled-Rennesson. Contribution à la reconnaissance des plans discrets. Technical report, Done at Loria Laboartory INRIA-Lorraine, April 2000.

[9] M.M. Mesmoudi and I. Debled-Rennesson. Sur la reconnaissance des plans discrets. In Colloque d'Analyse et Application. Mostaganem University, Oct 1999.

[10] J.P. Reveilles. Géométrie discrète, calculs en nombre entiers et algorithmique. Doctorat d'Etat thesis, Louis Pasteur University, Strasbourg, 1991.

[11] I. Stojmenović and R. Tosić. Digitaziation schemes and the recognition of digital straight lines, hyperplanes and flats in arbitrary dimensions. In Vision Geometry, Contemporay Mathematics series, volume 119, pages 197-212. AMS, Providence, RI, 1991.

[12] P. Veelaert. Digital planarity of rectangular surface segments. In IEEE Transactions on Pattern Analysis and Machine Intelligence, volume 16, pages 647-652, 1994.

[13] P. Veerlaert. On the flatness of digital hyperplanes, volume 3. Journal of Mathematical Imaging and Vision, 1993. 